\section{Freeze Fracturing of Hot Samples}

Thomas Rades, University of Otago, New Zealand

Liquid crystals are substances which combine properties of both liquids and solids. To investigate the structures and structural deiects of liquid crystals, freeze fracturing of these gel-like substances has been performed successfully for many years. In the TEM the structures of the different types of liquid crystals have been investigated and the results confirmed the earlier investigations using polarizing light microscopy. These TEM studies extended the knowledge of structures and structural defects of this fascinating state of matter. However, freeze fractures were only carried out on liquid crystals which form around or below room temperature.

Some liquid crystals form upon the addition of a solvent to a crystal (so called lyotropic liquid crystals) while others form upon heating of a crystalline structure (so called thermotropic liquid crystals). In the last few years we did some research on the pharmaceutical drug fenoprofen. This drug is able to form both types of liquid crystal. Lyotropic liquid crystals of fenoproten form at room temperature on the addition of water. The viscosity of this liquid crystal was low, comparable to that of a pharmaceutical cream, so there were few difficulties in performing freeze fracture. However, the thermotropic mesophases had a much higher viscosity and they only formed upon heating either the sodium salt of fenoprofen to temperatures above $105^{\circ} \mathrm{C}$.

To perform freeze fracture of the liquid crystal of the sodium salt we placed the crystalline drug on a copper sample holder and heated the sample and the upper part of the sample holder on a Kofler heater bench (C Reichert $\mathrm{AG}$ ) to various temperatures between $125 \mathrm{C}$ and $145^{\circ} \mathrm{C}$. (The temperature was chosen to be a bit higher than the transformation temperature.) The sample was allowed to equilibrate for 10 minutes at the final temperature.

A pair of tweezers were brought to the same temperature and were used to put the upper sample holder onto the liquid crystalline sample

Once the upper sample holder had been put onto the sample, the sample was transferred as fast as possible (using the same pair of tweezers)

Anyone Can Service Your SEM, But...

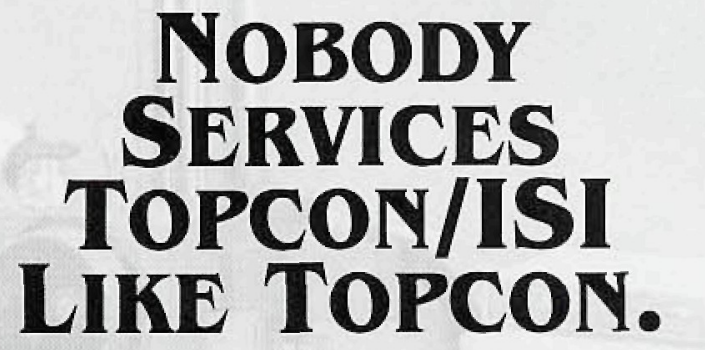

At Topcon we are committed to providing the best possible service for Topcon/ISI SEM's.

With our strong financial base and years of experience, we are able to offer our customers a wide variety of service options including:

- "FULL" Coverage Contracts

- Competitive Pricing

- Time and Materials Service

- Largest Stock of Topcon Factory Warranted Parts

- Nationwide Network of Highly Qualified Engineers

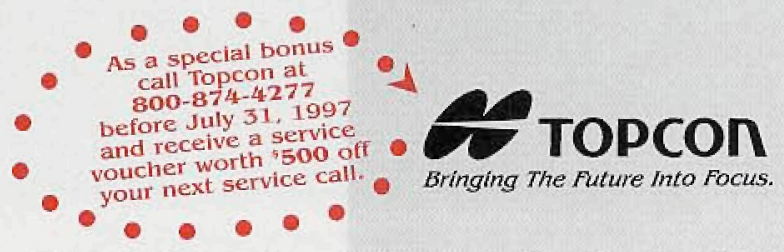

37 West Century Road - Paramus, New Jersey 07652 Phone: 800-874-4277 - Fax: 201-262-1504 to a maximally preheated Cryo-Jet (ca $90^{\circ} \mathrm{C}$ ) and was immediately frozen in liquid propane. To perform successful freezing, the amount of sample should be as small as possible. In fact, maybe one out of ten attempts was successful, while nine out of ten times the upper and lower sample holder separated. In later experiments we used nitrogen slush to perform the fast freezing with the same success, provided the pair of tweezers was heated to the same temperature as the sample. The freeze fracture was then carried out in a freeze-etch machine (BAF 400 , Balzers) at $-100^{\circ} \mathrm{C}$ and at a vacuum of $5 \times 10^{-6}$ bar

To produce a platinum/carbon replica, a $2 \mathrm{~nm}$ thick platinum shadowing layer was evaporated at a 45 degree angle and then a $20 \mathrm{~nm}$ thick layer of carbon was evaporated at a 90 degree angle.

The replicas were cleaned with chloroformimethanol $(1: 1(\mathrm{~V} / \mathrm{V})$ and water). Replicas on uncoated grids were viewed with a TEM (EM 300, Phillips) at $80 \mathrm{kV}$.

While the viscosity of the sodium salt of fenoproten was already high, the viscosity of the calcium salt in the liquid crystalline state was even higher, so that the liquid crystal in this case had a more solid than liquid appearance. We were not able to perform fast freezing when we heated the sample to temperatures only 10-30 $\mathrm{C}$ above the transition temperature. We noticed however that the viscosity of the sample decreased when the sample started to decompose at about $250^{\circ} \mathrm{C}$ From hot stage polarising light microscopy it was known that the liquid crystals were still present during the decomposition, so we performed the same procedure as described above to achieve the fast freezing. only this time with the sample heated to $250 \mathrm{C}$. Fast freezing was possible and the first TEM micrographs of this type of thermotropic liquid crystals could be made.

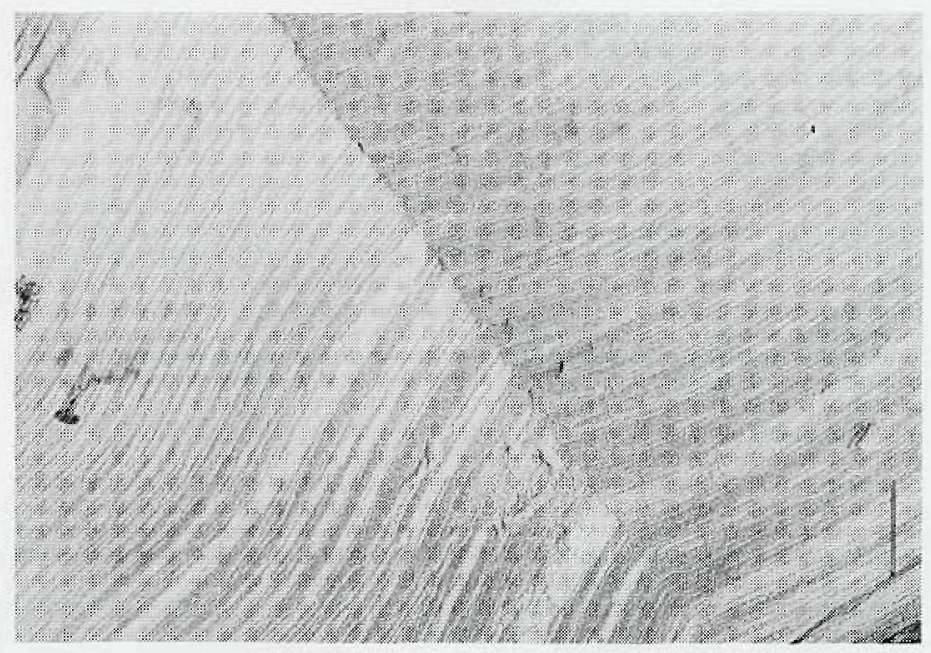

Figure 1: TEM micrograph of the smectic LC of fenoprofen sodium at $125^{\circ}$ C. Bar represents $200 \mathrm{~nm}$.

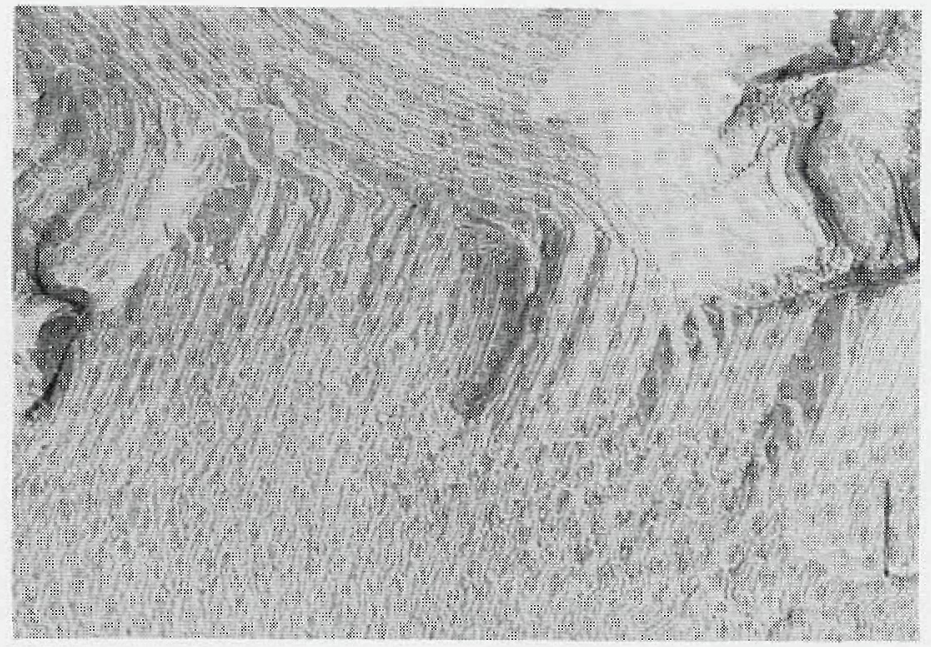

Figure 2: TEM micrograph of the reversed hexagonal LC of fenoprofen calcium at $250^{\circ} \mathrm{C}$. Bar represents $200 \mathrm{~nm}$.

Reprinted from Microscopy in Focus. the bulletin of the New Zealand Microscopy Society 


\section{The Series 5 \\ Robinson Detector}

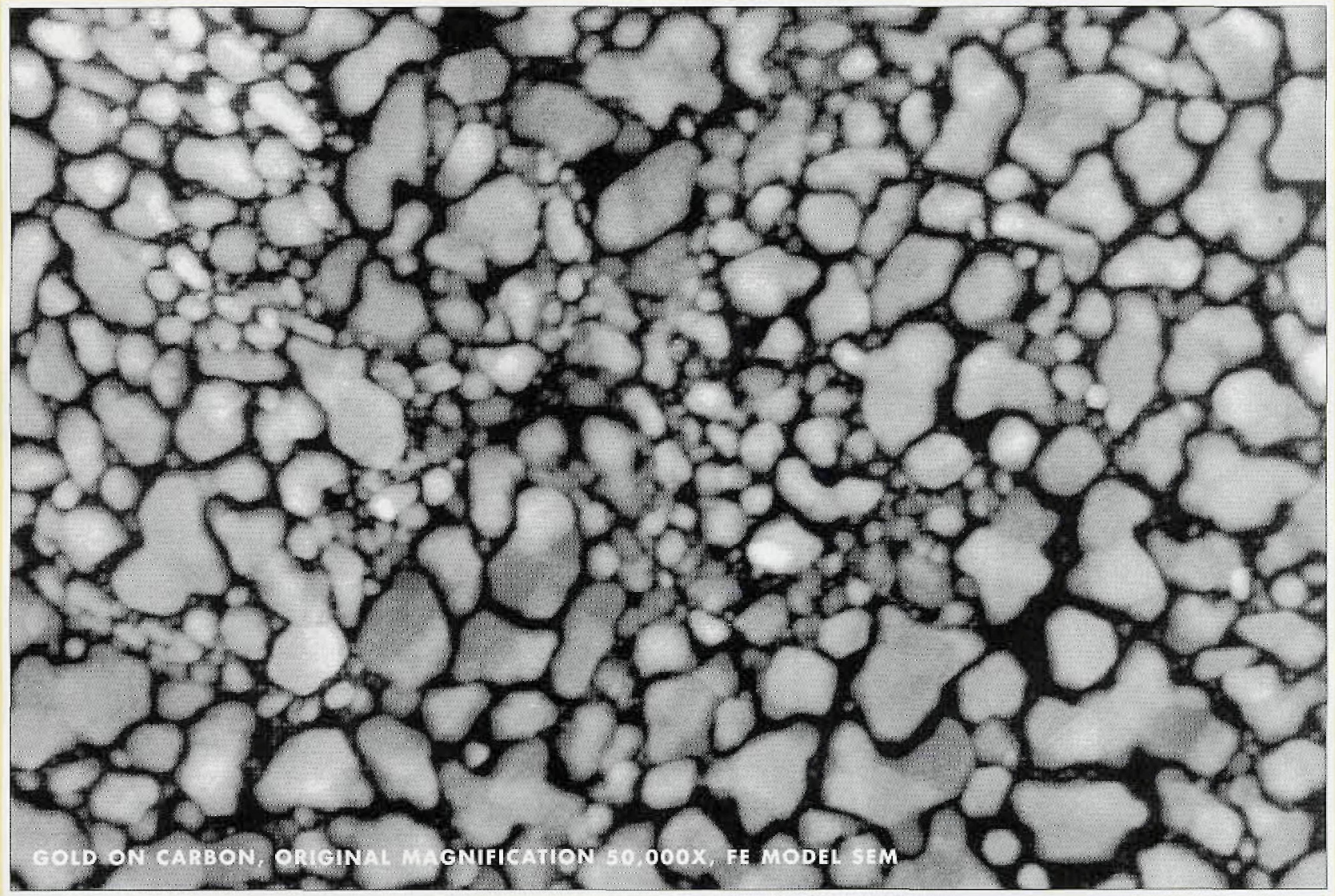

When image is everything,

only the best will do.

Whether you use conventional, fieldemission or environmental SEMs or defect review tools, the Series 5

Robinson BSE Detector delivers unprecedented performance. More signal, less noise, and outstanding resolution. Call us to find out more.

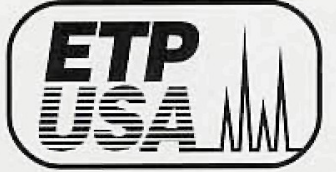

ELECTRON DETECTORS INC. 1650 Holmes Street, Livermore, CA 94550, USA.

Telephone: 1 (800) 8ETP USA, (510) 449-8534. Facsimile: (510) 449-8996.

Email: info@etp-usa.com Web: http://www.etp-usa.com 